DOI: $10.17516 / 1997-1397-20-13-1-58-70$

УДК 517.5

\title{
Berry Phase for Time-Dependent Coupled Harmonic Oscillators in the Noncommutative Phase Space via Path Integral Techniques
}

\author{
Leila Khiari* \\ LRPPS, University Kasdi Merbah \\ Ouargla, Algeria \\ Tahar Boudjedaa ${ }^{\dagger}$ \\ University of Jijel \\ Jijel, Algeria \\ Abdenacer Makhlouf ${ }^{\ddagger}$ \\ University of Haute Alsace \\ Mulhouse, France \\ Mohammed Tayeb Meftah ${ }^{\S}$ \\ LRPPS, University Kasdi Merbah \\ Ouargla, Algeria
}

Received 08.05.2019, received in revised form 15.10.2019, accepted 25.12.2019

Abstract. The purpose of this paper is the description of Berry's phase, in the Euclidean Path Integral formalism, for 2D quadratic system: two time dependent coupled harmonic oscillators. This treatment is achieved by using the adiabatic approximation in the commutative and noncommutative phase space.

Keywords: Berry's phase, noncommutative phase space, coupled oscillators.

Citation: L.Khiari, T.Boudjedaa, A.Makhlouf, M.Tayeb Meftah, Berry Phase for Time-Dependent Coupled Harmonic Oscillators in the Noncommutative Phase Space via Path Integral Techniques, J. Sib. Fed. Univ. Math. Phys., 2020, 13(1), 58-70.

DOI: $10.17516 / 1997-1397-20-13-1-58-70$.

\section{Introduction and preliminaries}

The classical geometry is based on the duality between the geometry and the commutative algebra. In commutative algebra, the product of two algebraic quantities is independent from the order. In Quantum Mechanics, following Heisenberg's viewpoint, the geometry of the states space describing a microscopic system, an atom for example, has a new property such as the momentum and the position are non-commuting operators [1-7]:

$$
\left[x_{i}, x_{j}\right]=i \theta_{i j}, \quad\left[p_{i}, p_{j}\right]=i \sigma_{i j}, \quad\left[x_{i}, p_{j}\right]=i \delta_{i j}
$$

The purpose of noncommutative geometry is to generalize the duality of space geometry [8-10] and algebra to the more general situation where the algebra is not commutative. This leads to

*leila.khiari2010@gmail.com

†boudjedaa@gmail.com

†abdenacer.makhlouf@uha.fr

$\S$ mewalid@yahoo.com

(c) Siberian Federal University. All rights reserved 
change two fundamental concepts of mathematics, those of space and symmetry and adjusts all the mathematical tools in these new paradigms.

The prime interest of the theory comes entirely from new and unexpected phenomena that have no counterpart in the case of commutative geometry. The commutative Riemannian geometry which provides a framework of general relativity was generalized by Einstein to "quantum" version.

The passage of the Riemann geometry to the noncommutative geometry [11] is the transition from the measurement of distances to the use of operators algebra. This gives a notion of a spectral nature of geometric space which is more flexible.

The noncommutative geometry treats both the noninteger dimension space [12,13], an infinite dimension space, especially "quantum" space and finally the space-time itself. If we take into account, not only the electromagnetic strength (which led to Poincaré, Einstein and Minkowski model of spacetime), but also the weak and strong forces, the use of the noncommutative spacetime properties becomes necessary.

Furthermore, Feynman paths integrals method encounters substantial difficulties when used in a noncommutative space because it is basically meaningless to talk about path in a noncommutative spacetime. Therefore the formulation of path integrals must done not in the space of noncommutative coordinates itself but in the space of noncommutative phase space (mixed space). This is required by the spirit of the Feynman path integrals construction.

Indeed, we consider a space-time $(2+1)$-dimensions, which can be easily generalized to higher dimensions. So, to conciliate our work in the canonical formalism, we used a full basis of commuting operators. We shall take as space, the configuration space $\left(x_{1}, p_{2}\right)$ built on commuting operators such that we avoid the noncommutativity.

In this paper we are mainly concerned with two coupled harmonic oscillators with arbitrary time dependent frequencies and masses leading to use some time-dependent transformations. The originality of this work, is the description of the system in the noncommutative mixed phase space by using the path integral techniques to extract the "Berry's phase". We recall that Berry's phase has attracted the attention of many physicists, it was first discovered in 1956, and rediscovered in 1984 by Berry who has published a paper [14] which has until now deeply influenced the physical community. Therein he considers cyclic evolutions of systems under special conditions, namely adiabatic ones. He finds that an additional phase factor occurs in contrast to the well known dynamical phase factor. This phenomenon can be described by "global change without local change". Berry points out the geometrical character of this phase which is not negligible because of its non integrable character [15]. This was not the first time such a phase factor appears, for instance, considerations of the Born-Oppenheimer approximation done by Mead and Truhlar in 1979 revealed also this additional phase factor but it had been neglected [16]. Berry showed that this was not correct because the phase is a gauge invariant and therefore can not be gauged away.

A brief outline of the present paper is as follows: in the next section, we give the construction of the path integral in the noncommutative phase space. In Section 3, we present two applications, the first one is the time dependent coupled harmonic oscillators in commutative phase space, the second application deals with the time-dependent coupled harmonic oscillators in noncommutative phase space. In each case, Berry's phase (geometric phase) was derived as well as the dynamic phase. A conclusion is provided in the last section.

\section{Path integral in noncommutative phase space}

In this section we must be concerned with Feynman's path integral formalism, which is described by a Hamiltonian $H(x, p)$ made up the cartesian coordinates $x_{i}$, and their canonically conjugate momenta $p_{j}$. Nevertheless, unlike the usual case, "coordinates and momenta" are 
assumed to obey the noncommutative rules.

$$
\left[x_{i}, x_{j}\right]=i \theta_{i j}, \quad\left[p_{i}, p_{j}\right]=i \boldsymbol{\Sigma}_{i j}, \quad\left[x_{i}, p_{j}\right]=i \delta_{i j},
$$

where $\boldsymbol{\Theta}$ and $\boldsymbol{\Sigma}$ are two-antisymmetric matrix such as $\boldsymbol{\Theta}_{12}=\theta$ and $\boldsymbol{\Sigma}_{12}=\sigma$. To these commutation relations correspond the deformed Poisson brackets in classical phase space defined as

$$
\left\{x_{i}, x_{j}\right\}=\theta_{i j}, \quad\left\{p_{i}, p_{j}\right\}=\boldsymbol{\Sigma}_{i j}, \quad\left\{x_{i}, p_{j}\right\}=\delta_{i j},
$$

where $\boldsymbol{\Theta}$ and $\boldsymbol{\Sigma}$ are noncommutative parameters.

Acting on the Heisenberg algebra (2), it is easy to found the Path integral in noncommutative phase space. But in this work, we propose to build the path integral while maintaining the spirit of Feynman's construction. On this basis, we choose the mixed phase space i.e. $Q^{T}=\left(x_{1}, p_{2}\right)$ and $P^{T}=\left(x_{2}, p_{1}\right)$.

A path integral formalism in noncommutative mixed coordinates is

$$
K_{\theta \sigma}\left(Q^{i}, Q^{f}, T\right)=\int D Q D P \exp \left[i \int\left[P J_{\theta \sigma}^{-1} \dot{Q}-H(Q, P)\right] d t\right]
$$

where $Q^{T}=\left(x_{1}, p_{2}\right), P^{T}=\left(x_{2}, p_{1}\right)$ and $\left(J_{\theta, \sigma}\right)_{i j}=\left(\begin{array}{cc}\theta & 1 \\ -1 & -\sigma\end{array}\right)$ is the symplectic form. For simplicity, the propagator (4), using linear canonical transformation which known as Bopp-shift in form matrix, may be written as

$$
K_{\theta \sigma}\left(Q^{i}, Q^{f}, T\right)=\int D Q D P \exp \left[i \int\left[P J_{0,0}^{-1} \dot{Q}-H_{\theta \sigma}(Q, \bar{A} P)\right] d t\right]
$$

where

$$
J_{0,0}=\left(\begin{array}{cc}
0 & 1 \\
-1 & 0
\end{array}\right), \quad \bar{A}=\left(\begin{array}{cc}
1 & \theta \\
\sigma & 1
\end{array}\right)
$$

As $\theta$ and $\sigma$ are very small parameters, we set $\bar{A}=1+\beta$, with $\beta=\left(\begin{array}{cc}0 & \theta \\ \sigma & 0\end{array}\right)$, then

$$
H_{\theta \sigma}(Q, \bar{A} P)=H(Q, P)+H_{\beta}(Q, P),
$$

with $H_{\beta}(Q, P)$ is now a small perturbation added to the Hamiltonian such that we can use Taylor's expansion of the Hamiltonian

$$
H_{\beta}(Q, P) \simeq(\beta P)^{T} \frac{\partial H(Q, P)}{\partial P}+\sum_{i j} \frac{1}{2 !}(\beta P)_{i}^{T}(\beta P)_{j} \frac{\partial^{2} H(Q, P)}{\partial P_{i} \partial P_{j}} .
$$

The Feynman's formalism for a general potential in noncommutative phase space is given by

$$
K_{\theta \sigma}\left(Q^{i}, Q^{f}, T\right)=\int D Q D P \exp \left[i \int d t\left(P J^{-1} \dot{Q}-H(Q, P)-H_{\beta}(Q, P)\right)\right] .
$$

The main goal of this paper is to find Berry phase of the 2-dimensional coupled harmonic oscillators in two cases the first one in commutative phase space, and the second case in noncommutative phase space, under the Euclidean path integral formalism. The original premise for Berry's phase is the adiabatic theorem of quantum mechanics [17], which deals with a system coupled to a slowly changing environment: the Hamiltonian system $H(t)$ varies adiabatically.

To extract the Berry's phase from the propagator (8), we follow the method used by Kashiwa to obtain the Berry's phase for one dimension harmonic oscillator, in which it summarizes as follows [18]. 
1. Consider the Euclidean kernel for the given Hamiltonian: $(t \rightarrow-i t)$ while keeping the external variables unchanged.

2. Examine the large $T$ limit of the kernel.

3. Find the imaginary part of $O(T)$ from the exponent of the kernel.

So, In the Euclidean space $(t \rightarrow-i t)$ and for adiabatic approximation, we set $s=\frac{t}{T}$ with $T$ very large, the $(8)$ is given by:

$$
K_{\theta \sigma}\left(Q^{i}, Q^{f}, T\right)=\int D Q D P \exp \left[T \int d s\left(\frac{i}{T} P J^{-1} \dot{Q}-H(Q, P)-H_{\beta}(Q, P)\right)\right],
$$

this latter is the Feynman's formalism in non-commutative phase space. where, we put $\theta, \sigma \rightarrow 0$, will return (9) to the commutative phase space (the usual phase space ), i.e, the (9) is given

$$
K\left(Q^{i}, Q^{f}, T\right)=\int D Q D P \exp \left[T \int d s\left(\frac{i}{T} P J^{-1} \dot{Q}-H(Q, P)\right)\right] .
$$

\subsection{Time-dependent coupled harmonic oscillators in commutative phase space}

Consider a pair of coupled general time-dependent oscillators with same frequencies and masses whose Hamiltonian in commutative phase space takes the form [21].

The quantum mechanical evolution of the system can be described by the Feynman propagator, in the mixed phase space $Q^{T}=\left(x_{1}, p_{2}\right)$ and $P^{T}=\left(x_{2}, p_{1}\right)$ (formulation of Feynman's path integral), which is defined formally by

$$
\begin{aligned}
& K\left(Q^{f}, Q^{i} ; t\right)=\int D Q D P= \\
&=\exp \left[\int\left(\left(P J_{0,0}^{-1} \dot{Q}-\frac{1}{2} P M(t) P-\frac{1}{2} Q W(t) Q-P \lambda(t) Q\right) d t\right] .\right.
\end{aligned}
$$

The matrices $M(t), W(t)$ and $\lambda(t)$ are time dependent functions given respectively by

$$
\left(\begin{array}{cc}
\mu_{1}(t) & 0 \\
0 & \mu_{2}(t)
\end{array}\right),\left(\begin{array}{cc}
\omega_{1}^{2}(t) & 0 \\
0 & \omega_{2}^{2}(t)
\end{array}\right),\left(\begin{array}{cc}
0 & \lambda_{2}(t) \\
\lambda_{1}(t) & 0
\end{array}\right)
$$

where

$$
\begin{array}{ll}
\mu_{1}(t)=m(t) \omega^{2}(t), & \mu_{2}(t)=\frac{1}{m(t)} \\
\omega_{1}(t)=\omega(t) \sqrt{m(t)}, & \omega_{2}(t)=\frac{1}{\sqrt{m(t)}} .
\end{array}
$$

Now, we follow the steps [18] that we have mentioned previously, we take $t \rightarrow-i t$, and in order to specify the adiabatic parameter $\frac{1}{T}$, we introduce a scaled times $s=t / T$ in (11), and after using change the variable $P \rightarrow J P$ on a level of Lagrange in (11), we get

$$
\begin{gathered}
K\left(Q^{i}, Q^{f}, T\right)=\int D Q D P= \\
=\exp \left[T \int\left(\frac{i}{T} P \dot{Q}-\frac{1}{2} P\left(J_{0,0} M J_{0,0}^{-1}\right) P-\frac{1}{2} Q W(s) Q-P(J \lambda(s)) Q\right) d s\right] . \\
-61-
\end{gathered}
$$


If we want to transform a Hamiltonton into a simpler and more convenient one, this is possible by using time-dependent canonical conversion, as the latter is very useful and effective in researching the properties of dynamical systems described by a time-dependent Hamiltonian. To simplify the Action given in (15), Let us introduce the canonical transformations which define the new phase space $(Q, P) \rightarrow(X, \Pi)[22-24]$ given by,

$$
\begin{aligned}
& \left(\begin{array}{l}
P_{1} \\
P_{2}
\end{array}\right)=\left(\begin{array}{cc}
\frac{1}{\sqrt{\mu_{2}(s)}} & 0 \\
0 & \frac{1}{\sqrt{\mu_{1}(s)}}
\end{array}\right)\left[\left(\begin{array}{c}
\Pi_{1} \\
\Pi_{2}
\end{array}\right)-\left(\begin{array}{cc}
\beta_{1}(s) & 0 \\
0 & \beta_{2}(s)
\end{array}\right)\left(\begin{array}{c}
X_{1} \\
X_{2}
\end{array}\right)\right], \\
& \left(\begin{array}{l}
Q_{1} \\
Q_{2}
\end{array}\right)=\left(\begin{array}{cc}
\sqrt{\mu_{2}(s)} & 0 \\
0 & \sqrt{X_{1}(s)}
\end{array}\right)\left(\begin{array}{c}
X_{2}
\end{array}\right),
\end{aligned}
$$

where the functions $\beta(s)$ can be conveniently chosen to make separation of variables straightforward possible. As a result of the transformation, and after using the gaussian integration over $\Pi$, one may write $(15)$ as

$$
\begin{aligned}
& K\left(Q_{j}^{f}, Q_{j}^{i} ; T\right)=\int D X \exp \frac{i[X(\beta(s)) X]_{0}^{T}}{2}= \\
&=\exp \left\{T \int\left(-\frac{1}{2 T^{2}} \dot{X} \dot{X}-\frac{1}{2}\left(\Omega_{1}^{c}\right)^{2}(s) X_{1}^{2}-\frac{1}{2}\left(\Omega_{2}^{c}\right)^{2}(s) X_{2}^{2}\right) d s\right\},
\end{aligned}
$$

where

$$
\left(\Omega_{(1,2)}^{c}(s)\right)^{2}=\mu_{(2,1)} \omega_{(1,2)}^{2}-\beta_{(1,2)}^{2}+\frac{i}{T} \mu_{(2,1)} \frac{d}{d s}\left(\frac{\beta_{(1,2)}}{\mu_{(2,1)}}\right)
$$

with

$$
\begin{aligned}
& \beta_{1}(s)=\lambda_{1}(s)-\frac{i}{2 T} \frac{\dot{\mu}_{2}(s)}{\mu_{2}(s)} \\
& \beta_{2}(s)=-\lambda_{2}(s)-\frac{i}{2 T} \frac{\dot{\mu}_{2}(s)}{\mu_{2}(s)} .
\end{aligned}
$$

Therefore, the propagator $K\left(Q_{j}^{f}, Q_{j}^{i} ; T\right)$ in (17) is now reduced to the sum of the propagators for two uncoupled general time-dependent oscillators of frequencies $\Omega_{(1,2)}^{c}(s)$, and same masses $m_{(1,2)}(s)=1$.

We know that the $W K B$-approximation, $\hbar$-expansion with $\hbar \rightarrow 0$ is almost equivalent to the adiabatic approximation, $\frac{1}{T}$-expansion with $T \rightarrow \infty$, It is known as well as that the easiest way to do the $W K B$ approximation is the path integral, this is confirmed by Kashiwa in his article [18], for this reason we see that the most appropriate way to perform an addiabatic approximation is the path integral.

It is clear that we can see that the adiabatic approximation in (15), so it is very easy to treat this latter directly by the semi-classical methods, in which case we resort to the Van Vleck-Pauli formula [18].

Hence, we have the Van Vleck-Pauli formula

$$
K\left(Q^{f}, Q^{i} ; T\right)=\sqrt{\operatorname{det}\left(\frac{i}{2 \pi} \frac{\partial^{2} S}{\partial X^{f} \partial X^{i}}\right)} \exp \left(-S\left(X^{f}, X^{i} ; T\right)\right),
$$

where, $S\left(X^{f}, X^{i} ; T\right)$ is the classical action defined by

$$
S\left(X^{f}, X^{i} ; T\right)=\frac{i[X(\beta(s)) X]_{0}^{T}}{2}+\int_{0}^{T} L\left(X^{f}, X^{i} ; T\right) d t,
$$


with

$$
L=\frac{-1}{2 T^{2}}\left(\frac{d X_{j}}{d s}\right)\left(\frac{d X_{j}}{d s}\right)-\frac{1}{2}\left(\Omega_{j}^{c}(s)\right)^{2}\left(X_{j}\right)^{2}, \quad j=1,2
$$

Now, that we have done all the steps described in APPENDIX, we get the propagator that accompanies this latter (23),

$$
K\left(Q^{f}, Q^{i} ; T\right)=\prod_{j=1}^{2} K\left(Q_{j}^{f}, Q_{j}^{i} ; T\right)
$$

with

$$
\begin{aligned}
K\left(Q_{j}^{f}, Q_{j}^{i} ; T\right) & =\left\{\prod_{j=1}^{2} \frac{\left(\mathbf{w}_{j}^{c}(T) \mathbf{w}_{j}^{c}(0)\right)^{\frac{1}{2}}}{2 \sinh \Theta_{j}(T)}\right\} \exp \left(-\frac{\sqrt{\mathbf{w}_{j}^{c}(T) \mathbf{w}_{j}^{c}(0)}}{2 \sinh \Theta_{j}(T)}=\right. \\
= & \left\{\left(\sqrt{\frac{\mathbf{w}_{j}^{c}(T)}{\mathbf{w}_{j}^{c}(0)}}\left(X_{j}^{f}\right)^{2}+\sqrt{\frac{\mathbf{w}_{j}^{c}(0)}{\mathbf{w}_{j}^{c}(T)}}\left(X_{j}^{i}\right)^{2}\right) \cosh \Theta_{j}(s)-2 X_{j}^{f} X_{j}^{i}\right\}= \\
& \left.=-\exp \frac{i[X \beta(s) X]_{0}^{T}}{2}\right)
\end{aligned}
$$

and where $X_{1}$ and $X_{2}$ are given by (16).

As it is known, informations on the ground state can be derived by setting $T \rightarrow \infty$ in (25). In fact, when we take this limit we obtain:

$$
\begin{aligned}
K\left(Q^{f}, Q^{i} ; T\right) \underset{T \rightarrow \infty}{\sim} & \left(\prod_{j=1}^{2}\left(\mathbf{w}_{j}^{c}(T) \mathbf{w}_{j}^{c}(0)\right)^{\frac{1}{4}}\right) e^{-\frac{1}{2} \Sigma_{j=1}^{2} \Theta_{j}(T)} \times \\
& \times \prod_{j=1}^{2} \exp \left\{\left(\left(\mathbf{w}_{j}^{c}(T)+i \beta(T)\right)\left(X_{j}^{f}\right)^{2}+\left(\mathbf{w}_{j}^{c}(0)-i \beta(0)\right)\left(X_{j}^{i}\right)^{2}\right)\right\} .
\end{aligned}
$$

In this formula, the imaginary part of $\Theta_{1}(T)$ and $\Theta_{2}(T)$ given by (63), corresponds to the Berry phase,

or

$$
\gamma_{(1,2)}(T)=\frac{1}{4} \int d t\left(\frac{\mu_{(2,1)}(t)}{\mathbf{w}_{(1,2)}(t)} \frac{d}{d t}\left(\frac{\lambda_{(1,2)}(t)}{\mu_{(2,1)}(t)}\right)\right)
$$

$$
\begin{aligned}
& \gamma_{1}(T)=\frac{1}{4} \int_{0}^{T} d t\left(\frac{1}{m(t) \sqrt{\omega^{2}(t)-\lambda_{1}^{2}(t)}} \frac{d}{d t}\left(m(t) \lambda_{1}(t)\right)\right) \\
& \gamma_{2}(T)=\frac{1}{4} \int_{0}^{T} d t\left(\frac{m(t) \omega^{2}(t)}{\sqrt{\omega^{2}(t)-\lambda_{2}^{2}(t)}} \frac{d}{d t}\left(\frac{\lambda_{2}(t)}{m(t) \omega^{2}(t)}\right)\right) .
\end{aligned}
$$

whereas the real parts of $\Theta_{1}(T)$ and $\Theta_{2}(T)$, correspond to the dynamical phase.

\subsection{Time-dependent coupled harmonic oscillator in noncommutative phase space}

To specify a particular system in the context of non-commutative quantum mechanics it is necessary to define the Hamiltonian $H_{\theta \sigma}=H_{n c}$. The latter must be chosen so that it is reduced to standard Hamiltonian. We consider a system of two coupled harmonic oscillators where the hamiltonian $H(t)$ is an explicit function of time, via the frequency $\omega(t)$ and the mass $m(t)$ which 
are functions of time. In the case of noncommutative phase space, this system is described by the following hamiltonian:

$$
\begin{aligned}
H_{\theta \sigma}\left(x_{1}, x_{2} ; p_{1}, p_{2}\right) \simeq & \left(\frac{1}{2 m(t)}+\frac{m(t) \theta^{2} \omega^{2}(t)}{2}\right) p_{1}^{2}+\frac{p_{2}^{2}}{2 m(t)}+\frac{1}{2} m(t) \omega^{2}(t) x_{1}^{2}+ \\
& +\left(\frac{m(t) \omega^{2}(t)}{2}+\frac{\sigma^{2}}{2 m(t)}\right) x_{2}^{2}+\lambda_{1}(t) \sigma x_{1} x_{2}+\lambda_{2} \theta p_{1} p_{2}+ \\
& +\left(\frac{\sigma}{m(t)}+\theta m(t) \omega^{2}(t)\right) p_{1} x_{2}+\lambda_{1}(t) p_{1} x_{1}+\lambda_{2}(t) p_{2} x_{2},
\end{aligned}
$$

where $\theta$ and $\sigma$ are the deformed parameters defined above in Section 2 .

We rewrite (30) using mixed coordinates $Q=\left(Q_{1}, Q_{2}\right)=\left(x_{1}, p_{2}\right)$ and $P=\left(P_{1}, P_{2}\right)=\left(x_{2}, p_{1}\right)$. Therefore the compact form of the above Hamiltonian is:

$$
H_{\theta \sigma}(Q, P)=\frac{1}{2} P M(t) P+\frac{1}{2} Q W(t) Q+P \lambda(t) Q
$$

where

$$
M(t)=\left(\begin{array}{cc}
\mu_{1}(t) & b(t) \\
b(t) & \mu_{2}(t)
\end{array}\right), W(t)=\left(\begin{array}{cc}
\omega_{1}^{2}(t) & 0 \\
0 & \omega_{2}^{2}(t)
\end{array}\right), \quad \lambda(t)=\left(\begin{array}{cc}
\lambda_{1}(t) \sigma & \lambda_{2}(t) \\
\lambda_{1}(t) & \lambda_{2}(t) \theta
\end{array}\right)
$$

with

$$
\begin{aligned}
\mu_{1}(t)= & \left(m(t) \omega^{2}(t)+\frac{\sigma^{2}}{m(t)}\right), \quad \mu_{2}(t)=\left(\frac{1}{m(t)}+m(t) \theta^{2} \omega^{2}(t)\right) \\
& b(t)=\left(\frac{\sigma}{m(t)}+\theta m(t) \omega^{2}(t)\right) \\
\omega_{1}(t)= & \sqrt{m(t)} \omega(t), \quad \omega_{2}(t)=\frac{1}{\sqrt{m(t)}} .
\end{aligned}
$$

We suggested setting $\theta=-\sigma$ and $m^{2}(t) \omega^{2}(t)=1$ to facilitate calculations, avoid repetition and reduce steps.

After this simplification, the matrix becomes as follows:

$$
M(t)=\left(\begin{array}{cc}
\mu_{1}(t)=\frac{1}{m(t)}\left(1+\sigma^{2}\right) & 0 \\
0 & \mu_{2}(t)=\frac{1}{m(t)}\left(1+\sigma^{2}\right)
\end{array}\right)
$$

The quantum mechanical evolution of the hamiltonian (30) can be described by the propagator, in the non-commutative phase space formulation of Feynman's path integral, which is defined by:

$$
\begin{aligned}
& K_{\theta \sigma}\left(Q^{f}, Q^{i} ; T\right)=\int D Q D P= \\
& \quad=\exp \left[T \int\left(\left(\frac{i}{T} P \dot{Q}-\frac{1}{2}\left(P\left(J^{-1} M(s) J\right) P+Q W(s) Q+2 P\left(J^{-1} \lambda(s)\right) Q\right)\right)\right) d s\right] .
\end{aligned}
$$

In this subsection, we are mainly interested to find the Berry's phase in the Euclidean path integral formalism in non-commutative phase space.

And to find the Berry phase in non-commutative phase space, we will follow the same steps we took in the case of the commutative phase space of two coupled harmonic oscillators, then 
we compare between the both applications in order to highlight the impact of the deformation parameters $(\theta, \sigma)$ on the hamiltonian.

We can see the path integral (37) is not trivial but can be important. In the case of making this equation (37) to a more easily form, it is useful to use the time-dependent canonical transformation. This transformation leads to an effective diagonal Hamiltonian in terms of noncommutative coordinates.

In order to remove the matrix $\left(J^{-1} M(s) J\right)$ and $\left(J^{-1} \lambda(s)\right)$, we can use the time-dependent canonical transformation $(P, Q) \rightarrow(\Pi, X)$ similar to that provided in [23] and [24]:

$$
\begin{aligned}
& \left(\begin{array}{l}
P_{1} \\
P_{2}
\end{array}\right)=\left(\begin{array}{cc}
\frac{1}{\sqrt{\mu}} & 0 \\
0 & \frac{1}{\sqrt{\mu}}
\end{array}\right)\left(\left(\begin{array}{l}
\Pi_{1} \\
\Pi_{2}
\end{array}\right)-\left(\begin{array}{ll}
\beta_{11}(s) & \beta_{12}(s) \\
\beta_{21}(s) & \beta_{22}(s)
\end{array}\right)\left(\begin{array}{c}
X_{1} \\
X_{2}
\end{array}\right)\right), \\
& \left(\begin{array}{l}
Q_{1} \\
Q_{2}
\end{array}\right)=\left(\begin{array}{cc}
\sqrt{\mu} & 0 \\
0 & \sqrt{\mu}
\end{array}\right)\left(\begin{array}{l}
X_{1} \\
X_{2}
\end{array}\right) .
\end{aligned}
$$

Hence, the new propagator for the system becomes

$$
\begin{aligned}
K_{\theta \sigma}\left(Q^{f}, Q^{i} ; T\right)=\int D X D \Pi \exp \left[\frac{i\left(X\left(\beta(s)+\beta^{t}(s)\right) X\right)}{2}\right]_{0}^{T} \times \\
\quad \times \exp (T) \int d s\left(\frac{i}{T} \Pi \dot{X}-\frac{1}{2} \Pi \Pi-\frac{1}{2} X \Omega^{n c}(s) X\right),
\end{aligned}
$$

where

$$
\begin{aligned}
\left(\Omega_{1}^{n c}\right)^{2} & =\mu \omega_{1}^{2}(t)+\lambda_{1}^{2}-\sigma^{2}\left(\lambda_{1} \lambda_{2}-\frac{1}{2} \lambda_{2}^{2}\right)-\frac{i}{T} \mu \frac{d}{d s}\left(\frac{\beta_{11}}{\mu}\right) \\
\left(\Omega_{2}^{n c}\right)^{2} & =\mu \omega_{2}^{2}(t)+\lambda_{2}^{2}-\sigma^{2}\left(\lambda_{1} \lambda_{2}-\frac{1}{2} \lambda_{1}^{2}\right)-\frac{i}{T} \mu \frac{d}{d s}\left(\frac{\beta_{22}}{\mu}\right) \\
\Omega_{3}^{n c} & =-\frac{i}{T} \mu \frac{d}{d s}\left(\frac{\beta_{12}}{\mu}\right)-\frac{i}{T} \mu \frac{d}{d s}\left(\frac{\beta_{21}}{\mu}\right)
\end{aligned}
$$

and the matrices elements of $\beta(s)$ are

$$
\begin{aligned}
\beta_{(11)}(s) & =\lambda_{(1,2)}-\frac{i}{2 T} \frac{\dot{\mu}}{\mu}, \\
\beta_{(22)}(s) & =-\lambda_{(1,2)}-\frac{i}{2 T} \frac{\dot{\mu}}{\mu}, \\
\beta_{(12,21)}(s) & =-\sigma \lambda_{(2,1)}
\end{aligned}
$$

in this case we took $\dot{\sigma}=0$, and in put $\lambda_{1}=-\lambda_{2}=\lambda$ the relations (41), (42) and (43) becomes:

$$
\left(\Omega_{(1,2)}^{n c}\right)^{2}(s)=\left(\mathbf{w}_{(1,2)}^{n c}\right)^{2}(s)-\frac{1}{T} \tilde{\omega}_{(1,2)}^{n c}(s),
$$

where

$$
\left(\mathbf{w}_{(1,2)}^{n c}\right)^{2}(s)=\left(\mathbf{w}_{(1,2)}^{c}\right)^{2}(s)-\frac{3 \sigma^{2}}{2} \lambda^{2}(s)
$$

and

$$
\tilde{\omega}_{(1,2)}^{n c}(s)=-i \mu \frac{d}{d s}\left(\frac{\lambda(s)}{\mu}\right),
$$


where $\Omega^{c}$ and $\Omega^{n c}$ are commutative and noncommutative frecuency.

The $\Pi$-integration in (40) is easily performed to give the new propagator:

$$
\begin{aligned}
K_{\theta \sigma}\left(Q^{f}, Q^{i} ; T\right)=\sqrt{2 \pi} \int D X \exp & {\left[\frac{i\left(X\left(\beta(s)+\beta^{t}(s)\right) X\right)}{2}\right]_{0}^{T}=} \\
& =\exp \left[T \int d s\left(\sum_{j=1,2}\left(\frac{-1}{2 T^{2}}\left(\dot{X}^{2}-\Omega_{j}^{2}(s) X_{j}^{2}\right)\right)\right)\right]
\end{aligned}
$$

The final expression of the propagator, for the system of two coupled harmonic oscillators in non-commutative phase space governed by the Hamiltonian (30), is given by

$$
\begin{aligned}
& K\left(Q_{j}^{f}, Q_{j}^{i} ;\right.T)=\left\{\prod_{j=1}^{2} \frac{\left(\mathbf{w}_{j}^{n c}(T) \mathbf{w}_{j}^{n c}(0)\right)^{\frac{1}{2}}}{2 \sinh \Theta_{j}(T)}\right\} \exp \left(-\frac{\sqrt{\mathbf{w}_{j}^{n c}(T) \mathbf{w}_{j}^{n c}(0)}}{2 \sinh \Theta_{j}(T)} \times\right. \\
& \times\left\{\left(\sqrt{\frac{\mathbf{w}_{j}^{n c}(T)}{\mathbf{w}_{j}^{n c}(0)}}\left(X_{j}^{f}\right)^{2}+\sqrt{\frac{\mathbf{w}_{j}^{n c}(0)}{\mathbf{w}_{j}^{n c}(T)}}\left(X_{j}^{i}\right)^{2}\right) \cosh \Theta_{j}(s)-2 X_{j}^{f} X_{j}^{i}\right\}- \\
&\left.-\exp \left[\frac{i\left(X\left(\beta(s)+\beta^{t}(s)\right) X^{\prime}\right)}{2}\right]_{0}^{T}\right),
\end{aligned}
$$

with

$$
\Theta_{(1,2)}(s)=T \int_{0}^{s} d \tau\left(\mathbf{w}_{(1,2)}^{n c}(\tau)-\frac{i}{2 T} \frac{\mu(\tau)}{\mathbf{w}_{(1,2)}^{n c}(s)} \frac{d}{d \tau}\left(\frac{\lambda(\tau)}{\mu(\tau)}\right)\right) .
$$

When we put $T \rightarrow \infty$ we have a real and imaginary part. This last corresponds to the Berry's phase which are as follows

$$
\gamma_{\theta \sigma}^{(1,2)}(s)=\frac{T}{4} \int d \tau\left(\frac{\mu(\tau) \frac{d}{d \tau}\left(\frac{\lambda(\tau)}{\mu(\tau)}\right)}{\sqrt{\left(\mathbf{w}_{(1,2)}^{c}\right)^{2}(s)-\frac{3 \sigma^{2}}{2} \lambda^{2}(s)}}\right) .
$$

Finally, we have found the Berry phase in the non-commutative state where depends this latter to the deformed parameters $\theta$ and $\sigma$, after we used a method of adiabatical approximation. In the case of $\theta=\sigma=0$, we can obtain exactly Berry's phase in the commutative phase space case.

\section{Conclusion}

In this paper we applied the path integral construction [1] in the noncommutative phase space, in which the structure of the phase space is deformed by introducing two deformation parameters $\theta$ and $\sigma$. We present an alternative treatment (via path integral formalism) for the problem of the coupled harmonic oscillators in two dimensions with time-dependent mass and frequency. We study two cases: the first one in commutative phase space and the second in noncommutative phase space. The treatment is based on the use of time-dependent canonical transformation and auxiliary time-dependent transformation by path integral techniques. To each canonical transformation correspond a new mass and a new frequency.

We know that the Berry phase is limited to the adiabatic approximation. We have calculated Berry phase in each case following the semi-classical solution via path integral. The result are two functions $\gamma^{(1)}(t)$ and $\gamma^{(2)}(t)$ in terms of the system parameters are $m(t), \omega(t), \lambda_{1}(t)$ and 
$\lambda_{2}(t)$ in the commutative case, but, in the case of the non-commutative phase space, the result are two functions also $\gamma_{\theta \sigma}^{(1)}(t)$ and $\gamma_{\theta \sigma}^{(2)}(t)$ in the terms of the system parameters $m(t), \omega(t)$, $\lambda_{1}(t)$ and $\lambda_{2}(t)$, in addition to the deformations parameters $\theta$ and $\sigma$, It is easy to see that we find the result of the Berry phase in commutative case in the limit $\theta, \sigma \rightarrow 0$.

\section{References}

[1] C.S.Acatrinei, J. Phys. A: Math. Gen., 37(2004), 1225-1230. DOI: $10.1088 / 0305-4470 / 37 / 4 / 010$

[2] C.S.Acatrinei, J. Phys. A: Math. Theor., 40(2007), F929-F933.

[3] B.Dragovish, Z.Rakic, Path Integral Approach to Noncommutative Quantum Mechanics, 2004, arXiv:hep-th/0401198v1.

[4] M.R.Douglas, N.A.Nekrasov, Rev. Mod. Phys., 73(2001), 977, arXiv:hep-th/0106048v4. DOI: 10.1103/RevModPhys.73.977

[5] M.Chaichian, A.Demichev, P.Presnajder, M.M.Sheikh-Jabbari, A.Tureanu, Phys. Lett. B, 527(2002), 149-154. DOI: 10.1016/S0370-2693(02)01176-0

[6] C.Duval, P.Horvathy, Phys. Lett. B, 479(2000), 284, DOI: 10.1016/S0370-2693(00)00341-5; C.Duval, P.Horvathy, J. Phys. A, 34(2001), 10097, DOI: 10.1088/0305-4470/34/47/314; P.Horvathy, Ann. Phys., 299(2002), 128, DOI: 10.1006/aphy.2002.6271.

[7] L.Gouba, A comparative review of four formulations of noncommutative quantum mechanics, 2016, arXiv:hep-th/1603.07176v2.

[8] A.Connes, M.R.Douglas, A.Schwarz, JHEP, (1998), DOI:10.1088/1126-6708/1998/02/003.

[9] M.Dubois-Violette, Noncommutative differential geometry, quantum mechanics and gauge Theory, In: 19th International Conference on Differential-geometric Methods in Theoretical Physics, C. Bartocci, U. Bruzzo, R. Cianci eds., Lecture Notes in Physics, Vol. 375, Rapallo, Italy, 1990, 13-24. DOI: 10.1007/3-540-53763-5

[10] V.P.Nair, A.P.Polychronakos, Phys. Lett. B, 505(2001), 267. DOI:10.1016/S0370-2693(01)00339-2

[11] A.Connes, Noncommutative Geometry, Academic Press, London, 1994.

[12] J.Bros, The Geometry of Relativistic Spacetime: from Euclid's Geometry to Minkowski's Spacetime. Séminaire Poincaré, 2005, 1-45.

[13] A.Smailagic, E.Spallucci, Journal of Physics A, 36(2003), no. 33, L467-L471.

[14] M.V.Berry, Proc. R. Soc. Lond. A, 392(1984), 45-57. DOI: 10.1098/rspa.1984.0023

[15] A.Shapere, F.Wilczek, Geometric phase in quantum Theory, Advanced Series In Mathematical Physics, Vol. 5, World Scientific, 1989.

[16] T.Pacher, C.A.Mead, L.S.Cederbaum, H.Koppel, J. Chem. Phys., 91(1989), 7057. DOI: $10.1063 / 1.457323$

[17] A.Messiah, Quantum Mechanics, Vol. II, ch. XVII, §13, New-York, Wiley and Sons, 1958. 
[18] T.Kashiwa, S.Nima, S.Sakoda, Vistas in Astronomy, 37(1993), 279-282.

DOI: $10.1016 / 0083-6656(93) 90048-O$

[19] D.C.Khandekar, S.V.Lawande, K.V.Bhagwat, J. Phys. A, 16(1983), 4209.

[20] B.Dragovish et al., Proc. Steklov Inst. Math., 265(2009), 82-91.

DOI: $10.1134 / \mathrm{S} 0081543809020072$

[21] C.Grosche, F.Steiner, Handbook of Feynman Path Integral, Springer tracts in modern physics, Vol. 145, 1998.

[22] J.Jing, You Cui, Zheng-Wen Long, Eur. Phys. J. C, 67(2010), 583-588.

DOI: $10.1140 / \mathrm{epjc} / \mathrm{s} 10052-010-1296-4$

[23] F.Benamira, L.Guechi, Czechoslovak Journal of Physics, 53(2003), no. 9, 717-725.

DOI: $10.1023 / \mathrm{A}: 1025931630841$

[24] S.Menouar, M.Maamache, J.Ryeol Choi, Annals of Physics, 325(2010), 1708-1719.

DOI: $10.1016 / \mathrm{j}$.aop.2010.04.011

[25] S.Zhang, J.R.Choi, C.I.Um, K.H.Yeon, Phys. Lett. A, 2002, 294-319.

\section{Appendix}

Rewrite equation (18) as follows

$$
\left(\Omega_{j}^{c}(s)\right)^{2}=\left(\mathbf{w}_{j}^{c}(s)\right)^{2}-\frac{1}{T} \tilde{w}_{j}(s), \quad j=1,2,
$$

where

$$
\mathbf{w}_{(1,2)}^{c}(s)=\left(\mu_{(2,1)} \omega_{(1,2)}^{2}-\lambda_{(1,2)}^{2}(s)\right)^{\frac{1}{2}}
$$

and

$$
\tilde{w}_{(1,2)}(s)=-i \mu_{(2,1)} \frac{d}{d s}\left(\frac{\beta_{(1,2)}}{\mu_{(2,1)}}\right) .
$$

From the Lagrangian (23) we extract the motion equations

$$
\left(\frac{d^{2} X_{(1,2)}}{d s^{2}}\right)-T^{2} \Omega_{(1,2)}^{2}(s) X_{(1,2)}=0
$$

The boundary conditions are

$$
X_{(j)}(0)=\frac{Q_{(1,2)}^{i}}{\sqrt{\mu_{(2,1)}(0)}}, \quad X_{(j)}(T)=\frac{Q_{(1,2)}^{f}}{\sqrt{\mu_{(2,1)}(T)}}, \quad j=1,2 .
$$

Now the only task needed here is to find a classical solution $X_{(j)}^{c}$ of Equation (57), we consider for this the two Ansatz

$$
X_{(1,2)}=e^{T \int_{0}^{s} d \sigma \rho^{(1,2)}(\sigma)} a_{(1,2)} ; \quad \rho^{(1,2)}(s)=\sum_{n=0}^{\infty} \rho_{n}^{(1,2)}(0)\left(\frac{1}{T}\right)^{n}
$$

where $a_{(1,2)}$ is a given constant. Substituting equation (59) into (57), and using (54), lead to

$$
T^{2}\left(\rho_{0}^{(1,2)}\right)^{2}+T \dot{\rho}_{0}^{(1,2)}+\left(\rho_{1}^{(1,2)}\right)^{2}-T^{2}\left(\mathbf{w}_{(1,2)}^{c}(s)\right)^{2}-T \tilde{w}_{(1,2)}(s)=0 .
$$


By identification of coefficients with respect to $T$, and by restricting to $O\left(T^{2}\right)$ and $O(T)$, we obtain

$$
\left\{\begin{array}{cr}
\rho_{0}^{(1,2)}= \pm \mathbf{w}_{(1,2)}^{c}(s) & O\left(T^{2}\right) \\
\rho_{1}^{(1,2)}=\frac{-\dot{\rho}_{0}^{(1,2)}+\tilde{w}_{(1,2)}(s)}{2 \rho_{0}^{(1,2)}} & O(T) .
\end{array}\right.
$$

Finally, by taking into account the boundary condition (58), we get

$$
\begin{array}{r}
X_{(1,2)}(s)=\frac{1}{\sqrt{\mathbf{w}_{(1,2)}^{c}(s)} \sinh \Theta_{(1,2)}(T)}\left\{\sqrt{\frac{\mathbf{w}_{(1,2)}^{c}(T)}{\mu_{(1,2)}(T)}} Q_{(1,2)}^{f} \sinh \Theta_{12}(s)+\right. \\
\left.\quad+\sqrt{\frac{\mathbf{w}_{(1,2)}^{c}(0)}{\mu_{(1,2)}(0)}} Q_{(1,2)}^{i} \sinh \bar{\Theta}_{(1,2)}(s)\right\} \times\left\{1+O\left(\frac{1}{T}\right)\right\},
\end{array}
$$

where

$$
\Theta_{(1,2)}(s)=T \int_{0}^{s} d \tau\left(\mathbf{w}_{(1,2)}^{c}(\tau)-\frac{i}{2 T} \frac{\mu_{(2,1)}(\tau)}{\mathbf{w}_{(1,2)}^{c}(\tau)} \frac{d}{d \tau}\left(\frac{\lambda_{(1,2)}(\tau)}{\mu_{(2,1)}(\tau)}\right)\right)
$$

and $\bar{\Theta}_{j}(s)=\Theta_{j}(T)-\Theta_{j}(s)$. The action $S\left(X^{f}, X^{i} ; T\right)$ could be computed using the last solution. Indeed, integration by parts in the kinetic term of the action and the use of the motion equation (57) give

$$
\begin{aligned}
S\left(X^{f}, X^{i} ; T\right)=\frac{i[X \beta(s) X]_{0}^{T}}{2}+\int_{0}^{T} & \left(\frac{-1}{2 T^{2}}\left(\frac{d X_{j}}{d s}\right)\left(\frac{d X_{j}}{d s}\right)-\frac{1}{2} \Omega_{j}^{2}(s) X_{j}^{2}\right) d s= \\
& =\frac{i[X \beta(s) X]_{0}^{T}}{2}+\frac{1}{2 T}\left[X_{(1,2)} \frac{d X_{(1,2)}}{d s}\right]_{0}^{T} \simeq S_{1}+S_{2} .
\end{aligned}
$$

Straightforward calculation provides

$$
\begin{aligned}
& S_{2}=\frac{\sqrt{\mathbf{w}_{(1,2)}^{c}(T) \mathbf{w}_{(1,2)}^{c}(0)}}{2 \sinh \Theta_{(1,2)}(T)} \times \\
& \times\left\{\left(\sqrt{\frac{\mathbf{w}_{(1,2)}^{c}(T)}{\mathbf{w}_{(1,2)}^{c}(0)}}\left(X_{(1,2)}^{f}\right)^{2}+\sqrt{\frac{\mathbf{w}_{(1,2)}^{c}(0)}{\mathbf{w}_{(1,2)}^{c}(T)}}\left(X_{(1,2)}^{i}\right)^{2}\right) \operatorname{coth} \Theta_{(1,2)}(T)-2 X_{(1,2)}^{f} X_{(1,2)}^{i}\right\},
\end{aligned}
$$

yielding the determinant to

$$
\sqrt{\operatorname{det}\left(\frac{i}{2 \pi} \frac{\partial^{2} S}{\partial X_{(1,2)}^{f} \partial X_{(1,2)}^{i}}\right)}=\frac{\sqrt{\mathbf{w}_{(1,2)}^{c}(T) \mathbf{w}_{(1,2)}^{c}(0)}}{2 \sinh \Theta_{(1,2)}(T)} \cdot \sqrt{\frac{\mathbf{w}_{(1,2)}^{c}(T) \mathbf{w}_{(1,2)}^{c}(0)}{\mu_{(1,2)}(0) \mu_{(1,2)}(T)}}
$$




\title{
Фаза Берри для нестационарных связанных гармонических осцилляторов в некоммутативном фазовом пространстве с помощью методов интеграла по траектории
}

\author{
Лейла Хиари \\ Университет Касди Мербах \\ Уаргла, Алжир \\ Тахар Буджедаа \\ Университет Джиджель \\ Джиджель, Алжир \\ Абденасер Махлуф \\ Университет Высшего Эльзаса \\ Мюлуз, Франция \\ Мохамед Таеб Мефта \\ Университет Касди Мербах \\ Уаргла, Алжир
}

\begin{abstract}
Аннотация. Целью данной работы является описание фазы Берри в формализме евклидова интеграла по путям для двумерной квадратичной системы: двух связанных во времени гармонических осцилляторов. Эта обработка достигается с помощью адиабатического приближения в коммутативном и некоммутативном фазовом пространстве.

Ключевые слова: фаза Берри, некоммутативное фазовое пространство, связанные осцилляторы.
\end{abstract}

